\title{
ESTUDIO COMPARATIVO DE LOS DERMATOGLIFOS DIGITALES DE LOS ZOQUES Y OTROS GRUPOS INDÍGENAS DEL SURESTE DE MÉXICO *
}

\author{
Carlos Serrano Sánchez \\ Centro de Estudios Mayas UNAM.
}

Los zoques constituyen un importante grupo indígena asentado principalmente en la región centro-occidental del Estado de Chiapas (Fig. 1), aunque abarca también algunos municipios de Tabasco y Oaxaca. El número actual de hablantes de la lengua zoque sobrepasa los 20,000 sujetos (Thomas, 1974), y la vitalidad cultural del grupo, a pesar de su declinación paulatina, puede considerarse aún. muy fuerte.

Sin embargo, como se ha señalado recientemente (Navarrete, 1970), los zoques permanecen prácticamente desconocidos en sus diversos aspectos antropológicos; pero la carencia de información sobre este grupo quizá sea más patente en el campo de la Antropología Física; se pueden citar únicamente dos trabajos realizados sobre los zoques: el de Starr, que data de principios de siglo (1902), sobre características antropométricas, y el de Matson y. Swanson, sobre tipología sanguínea, efectuado en 1960 y al parecer aún inédito (alunos datos publicados en Matson, 1970).

Nuestro trabajo corresponde pues, a un propósito de ampliar este parco conocimiento de los zoques en sus características somáticas, que pueda conducir eventualmente a la comprensión de las relaciones biológicas de este grupo con otros pueblos indígenas mesoamericanos, especialmente los de la zona maya, de los cuales son vecinos.

Con este interés examinaremos aquí algunas características dermopapilares de los zoques, comparativamente a las que se encuentran en otros grupos del sureste de México.

Desde el punto de vista lingüístico, el zoque se clasifica dentro

* Trabajo presentado en el XLI Congreso Internacional de Americanistas, México, 1974. 


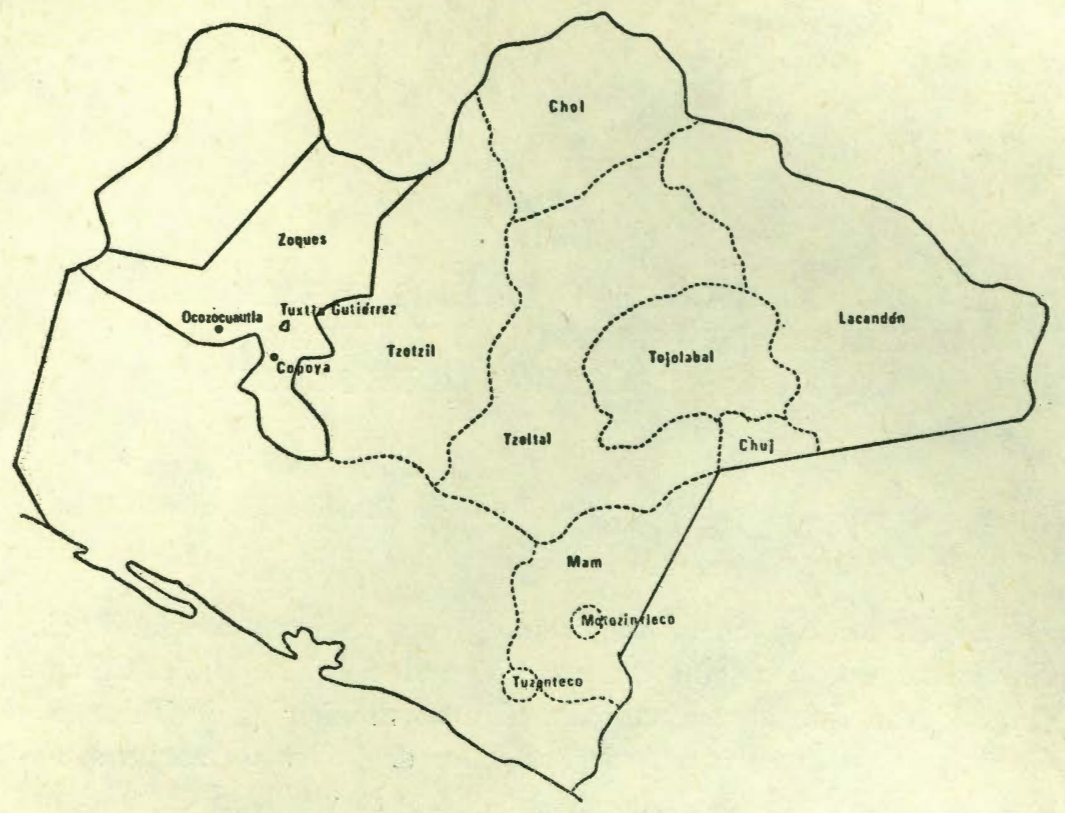

Figura 1. Distribución geográfica aproximada de los Zoques y grupos Mayances vecinos en el Estado de Chiapas y ubicación de las localidades estudiadas.

del grupo zoque-mixe, a su vez perteneciente a la gran familia macro-maya. Los cálculos glotocronológicos establecen una separación de esta lengua de los mayas en un momento que data de hace más de 3,000 años '(Swadesh, 1966). Podemos plantearnos la interrogante de si este grupo presenta un parentesco biológico con otros pueblos mayances, de la misma manera (y hasta qué grado en caso de respuesta afirmativa), que existe una relación lingüística, aunque lejana, con las poblaciones de habla maya.

El interés antropológico de los dermatotoglifos, que son los diseños o dibujos formados por las crestas papilares en las yemas de los dedos, palmas de las manos y plantas de los pies, ha sido enfatizado por diversos autores (Rife, 1935, Pons, 1963), y pueden considerarse una fuente de información de primera importancia (Newman, 1970) para el análisis de la historia racial de los indígenas americanos.

Consideramos que el análisis dermatoglífico, en razón del determinismo genético y gran estabilidad de este tipo de caracteres, puede proporcionar elementos interpretables en función del problema que se plantea. 


\section{Material y métodos}

Las muestras de impresiones dígito-palmares que se examinan en este trabajo se recogieron a principios de 1973, en dos localidades cercanas a Tuxtla Gutiérrez, Chis. La primera en Copoya, pueblo indígena situado a unos $10 \mathrm{Kms}$. al sur de la capital del estado, cuyo acceso se logra a través de una carretera de reciente construcción. Se trata de una comunidad bastante endogámica, con una tradición cultural muy fuerte. La mayoría de los adultos hablan regularmente su lengua y conservan su indumentaria tradicional.

El otro sitio en que se trabajó es Ocozocuautla, localidad urbana situada a unos $35 \mathrm{Kms}$. al oeste de Tuxtla Gutiérrez. Los individuos examinados en este último lugar son los habitantes del barrio "Unión Hidalgo", llamado también "El Paraje", que constituye la sección indígena de la ciudad. Se trata, en efecto, de familias que conservan sus propias características culturales a pesar de su vecindad con la población mestiza.

Se examinaron 125 sujetos en Copoya ( 74 hombres y 51 mujeres) y 48 en Ocozocuautla ( 21 hombres y 27 mujeres).

Las impresiones dermopapilares se obtuvieron con la técnica comúnmente utilizada de aplicar tinta sobre las yemas de los dedos y palma de la mano y recoger las huellas sobre papel. La interpretación de los caracteres dermatoglíficos se ha basado en las indicaciones de Cummins y Midlo (1961).

En este trabajo los datos estudiados se presentan como resultados preliminares y se refieren a las siguientes características dermatoglíficas:

a) Diseños digitales (frecuencias globales y distribución por dígitos, sexo y mano).

b) Porcentajes de deltas (también llamado "Indice de Cummins").1

La clasificación utilizada de los diseños digitales es la de Arcos, Presillas y Torbellinos; en el caso de las presillas se ha considerado su orientación radial o cubital.

1 El porcentaje de deltas es una cifra que representa la intensidad de desarrollo o de complejidad de los diseños digitales, de acuerdo al número de deltas o triradios (puntos donde convergen tres crestas papilares). Los arcos no tienen triradios, valor igual a 0 ; las presillas tienen uno, valor de 1 , y los torbellinos poseen dos triradios, valor igual a 2. Cada individuo presenta así un valor numérico según el tipo de dermatoglifos existentes en sus diez dedos, igual a la suma de los valores de cada dedo. Se puede entonces obtener el promedio de deltas o triradios que caracteriza a la población. 
Cuadro 1

DISTRIBUGIÓN PORCENTUAL DE DERMATOGLIFOS DIGITALES

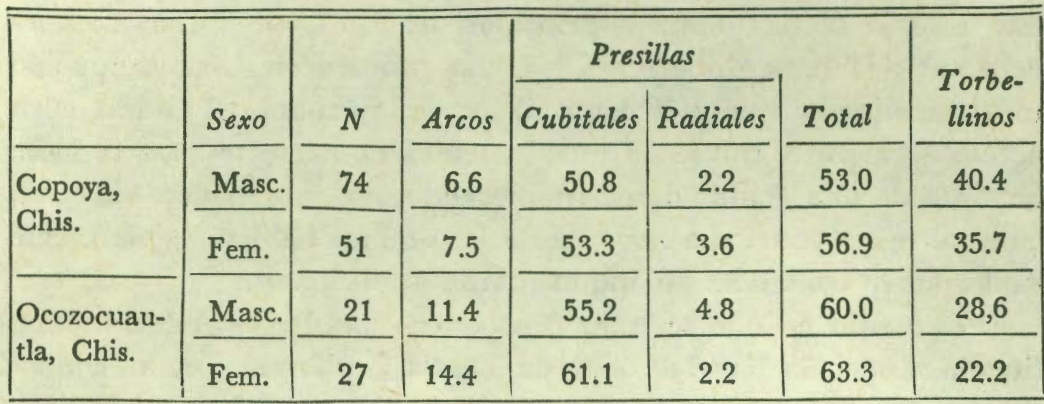

\section{RESULTADOS}

Frecuencias globales de dermatoglifos digitales

Las frecuencias de los diferentes tipos de diseños digitales entre los zoques de Copoya y Ocozocuautla se presentan en el cuadro 1. En ambas muestras el patrón dermatoglífico más común son las presillas, que predominan claramente sobre los torbellinos, como se observa generalmente en las poblaciones indígenas americanas. En cuanto a los arcos, su frecuencia es relativamente elevada, si se tiene en cuenta que en la mayoría de esos grupos este tipo de diseño alcanza sólo alrededor del $3 \%$.

Por otro lado, a pesar de las mismas tendencias dermatoglíficas en ambas muestras, se constata una clara diferencia en los porcentajes de los diseños digitales: una mayor incidencia de las presillas en detrimento de la frecuencia de torbellinos en la serie de Ocozocuautla. Entre los zoques de Copoya, en cambio, los torbellinos pre-

\section{Gúaro 2}

INDIGE DE GUMMINS EN DOS MUESTRAS ZOQUES DE CHIAPAS Y RESULTADOS DE LA PRUEBA t

\begin{tabular}{|c|c|c|c|c|c|c|}
\hline Sexo & Localidad & $n$ & $m$ & $s$ & $t$ & $P$ \\
\hline Masc. & $\begin{array}{l}\text { Copoya } \\
\text { Ocozocuautla }\end{array}$ & $\begin{array}{l}74 \\
21\end{array}$ & $\begin{array}{l}13.37 \\
11.71\end{array}$ & $\begin{array}{l}3.57 \\
3.61\end{array}$ & 1.88 & .1 \\
\hline Fem. & $\begin{array}{l}\text { Copoya } \\
\text { Ocozocuautla }\end{array}$ & $\begin{array}{l}51 \\
27\end{array}$ & $\begin{array}{l}12.82 \\
10.77\end{array}$ & $\begin{array}{l}3.76 \\
3.57\end{array}$ & 2.38 & .05 \\
\hline
\end{tabular}


sentan una frecuencia, en el sexo masculino, de alrededor del $40 \%$ (12\% más que en Ocozocuautla).

Estas diferencias entre las series de ambas localidades, en cada sexo, se reflejan claramente en el cálculo del porcentape de deltas ("pattern intensity index"), cuyos valores medios aparecen en el cuadro 2. La aplicación de la prueba $\mathrm{t}$ (Student) indica que la diferencia es significativa estadísticamente al nivel de .05 en el sexo femenino. Este fenómeno podría interpretarse en función de un mayor mestizaje biológico en los sujetos del barrio zoque de Ocozocuautla (a pesar de sus características culturales indígenas), que se expresa en un aumento del porcentaje de las presillas y reducción del de los torbellinos. Las cifras obtenidas en la población de este último sitio se aproxima así a las obtenidas en una muestra de la población urbana de la ciudad de México (Zavala et al., 1969) (cuadro 6).

Por esta razón se examinará aquí solamente la muestra de Copoya, como representativa de la población zoque.

Distribución de dermatoglifos en los diferentes dígitos

En el cuadro 3 se muestra el ordenamiento de los dedos en sentido decreciente, de acuerdo a la frecuencia de los distintos tipos de diseños.

\section{Cuadro 3}

ORDENAMIENTO DECRECIENTE DE DIGITOS SEGỨN LA FRECUENCIA DE TIPO DE DISENOO. ZOQUES DE COPOYA, CHIS.

\begin{tabular}{|c|c|c|}
\hline $\begin{array}{l}\text { Patrón } \\
\text { Dermatoglifico }\end{array}$ & Sexo & Dedos \\
\hline $\operatorname{Arcos}$ & $\begin{array}{l}\text { Masculino } \\
\text { Femenino }\end{array}$ & $\begin{array}{l}\text { II, III, I, IV, V. } \\
\text { II, III, I, IV. }\end{array}$ \\
\hline $\begin{array}{l}\text { Presillas } \\
\text { Cubitales }\end{array}$ & $\begin{array}{l}\text { Masculino } \\
\text { Femenino }\end{array}$ & $\begin{array}{l}\text { V, III, IV, II, I. } \\
\text { V, III, IV, I, II, }\end{array}$ \\
\hline $\begin{array}{l}\text { Presillas } \\
\text { Radiales }\end{array}$ & $\begin{array}{l}\text { Masculino } \\
\text { Femenino }\end{array}$ & $\begin{array}{l}\text { II, I, III, } \\
\text { II, III, IV. }\end{array}$ \\
\hline Torbellinos & $\begin{array}{l}\text { Masculino } \\
\text { Femenino }\end{array}$ & $\begin{array}{l}\text { I, IV, II, III, V. } \\
\text { I, IV, II, V, III. }\end{array}$ \\
\hline
\end{tabular}


Guadro 4

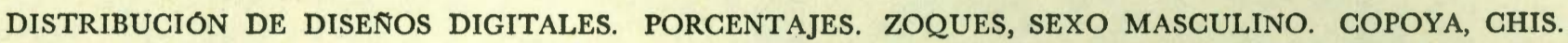

\begin{tabular}{|c|c|c|c|c|c|c|}
\hline \multirow[b]{2}{*}{ Dígito } & \multirow[b]{2}{*}{ Lado } & \multirow[b]{2}{*}{ Arcos } & \multicolumn{3}{|c|}{ Presillas } & \multirow[b]{2}{*}{ Torbellinos } \\
\hline & & & Cubitales & Radiales & Total & \\
\hline \multirow{3}{*}{ I } & Der. & 1.35 & 32.43 & - & 32.43 & 66.22 \\
\hline & Izq. & 1.35 & 40.54 & - & 40.54 & 58.11 \\
\hline & $\mathrm{D}+\mathrm{I}$. & 1.35 & 36.49 & - & 36.49 & 62.16 \\
\hline \multirow{3}{*}{ II } & Der. & 14.86 & 28.38 & 8.11 & 36.49 & 48.65 \\
\hline & Izq. & 14.86 & 31.08 & 10.81 & 41.89 & 43.24 \\
\hline & $\mathrm{D}+\mathrm{I}$ & 14.86 & 29.73 & 9.46 & 39.19 & 45.95 \\
\hline \multirow{3}{*}{ III } & Der. & 8.11 & 74.32 & 1.35 & 75.68 & 16.22 \\
\hline & Izq. & 12.16 & 67.57 & - & 67.57 & 20.27 \\
\hline & $\mathrm{D}+\mathrm{I}$ & 10.14 & 70.95 & 0.68 & 71.62 & 18.24 \\
\hline \multirow{3}{*}{ IV } & Der. & 4.05 & 36.49 & 1.35 & 37.84 & 58.11 \\
\hline & Izq. & 5.41 & 40.54 & - & 40.54 & 54.05 \\
\hline & $\mathrm{D}+\mathrm{I}$ & 4.73 & 38.51 & 0.68 & 39.19 & 56.08 \\
\hline \multirow{3}{*}{ V } & Der. & 1.35 & 81.08 & - & 81.08 & 17.57 \\
\hline & Izq. & 2.70 & 75.68 & - & 75.68 & 21.62 \\
\hline & $\mathrm{D}+\mathrm{I}$ & 2.03 & 78.38 & - & 78.38 & 19.59 \\
\hline \multirow{3}{*}{ I.V } & Der. & 5.95 & 50.54 & 2.16 & 52.70 & 41.35 \\
\hline & Izq. & 7.30 & 51.08 & 2.16 & 53.24 & 39.46 \\
\hline & $\mathrm{D}+\mathrm{I}$. & 6.62 & 50.81 & 2.16 & 52.97 & 40.41 \\
\hline
\end{tabular}

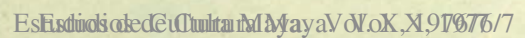

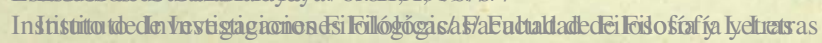

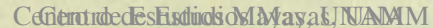

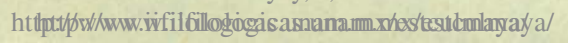


Cunadro 5

DISTRIBUCIÓN DE DISENOS DIGITALES. PORGENTAJES. ZOQUES, SEXO FEMENINO. COPOYA, CHIS.

\begin{tabular}{|c|c|c|c|c|c|c|}
\hline \multirow[b]{2}{*}{ Dígito } & \multirow[b]{2}{*}{ Lado } & \multirow[b]{2}{*}{ Arcos } & \multicolumn{3}{|c|}{ Presillas } & \multirow[b]{2}{*}{ Torbellinos } \\
\hline & & & Cubitales & Radiales & Total & \\
\hline \multirow{3}{*}{ I } & Der. & 5.88 & 29.41 & 5.88 & 35.29 & 58.82 \\
\hline & Izq. & 5.88 & 37.25 & 3.92 & 41.18 & 52.94 \\
\hline & $\mathrm{D}+\mathrm{I}$ & 5.88 & 33.33 & 4.90 & 38.24 & 55.88 \\
\hline \multirow{3}{*}{ II } & Der. & 15.69 & 39.22 & 5.88 & 49.02 & 35.29 \\
\hline & Izq. & 21.57 & 31.37 & 11.76 & 43.14 & 35.29 \\
\hline & $\mathrm{D}+\mathrm{I}$ & 18.63 & 35.29 & 10.78 & 46.08 & 35.29 \\
\hline 2 & Der. & 3.92 & 80.39 & - & 80.39 & 15.69 \\
\hline \multirow[t]{2}{*}{ III } & Izq. & 11.76 & 60.78 & 3.92 & 64.71 & 23.53 \\
\hline & $\mathrm{D}+\mathrm{I}$ & 7.84 & 70.59 & 1.96 & 72.55 & 19.61 \\
\hline \multirow{3}{*}{ IV } & Der. & 1.96 & 50.98 & - & 50.98 & 47.06 \\
\hline & Izg. & 7.84 & 39.22 & 5 & 39.22 & 52.94 \\
\hline & $\mathrm{D}+\mathrm{I}$ & 4.90 & 45.10 & - & 45.10 & 30.39 \\
\hline \multirow{3}{*}{ V } & Der. & - & 80.39 & - & 80.39 & 19.61 \\
\hline & Izq. & - & 84.31 & - & 84.31 & 15.69 \\
\hline & $\mathrm{D}+\mathrm{I}$ & $=$ & 82.35 & - & 82.35 & 17.65 \\
\hline \multirow{3}{*}{ I.V } & Der. & 5.49 & 56.08 & 3.14 & 59.22 & 35.29 \\
\hline & Izq. & 9.41 & 50.59 & 3.92 & 54.51 & 36.08 \\
\hline & $D+1$ & 7.45 & 53.33 & 3.53 & 56.86 & 35.69 \\
\hline
\end{tabular}


Se podrá observar que en ambos sexos, los arcos son más frecuentes en los dedos índice, medio y pulgar, las presillas cubitales se presentan con frecuencia mayor en el meñique, medio y anular; las presillas radiales, en el dedo índice, y los torbellinos, finalmente, en el pulgar, anular e índice.

Este ordenamiento de diseños digitales en los diferentes dedos corresponde al que generalmente se encuentra en los diversos grupos humanos.

Distribución de diseños digitales en función del sexo y la mano

Las frecuencias porcentuales de los dermatoglifos digitales en la muestra de Copoya, teniendo en cuenta cada uno de los dedos en ambas manos, 'se presentan en los cuadros 4 y 5 , para el sexo masculino y femenino, respectivamente.

Conforme a la observación más corriente, se aprecia una frecuencia relativamente mayor de presillas en el sexo femenino y de torbellinos en el masculino. Los arcos, como es también más común, predominan relativamente en esta serie entre las mujeres. Esta situación reproduce en cierto modo la distribución, bien conocida, de los dermatoglifos de acuerdo al lado: la mano derecha presenta mayor número de torbellinos (carácter masculino) y la mano izquierda mayor número de presillas (carácter femenino).

\section{Comparación con otras poblaciones indigenas}

Finalmente, interesa comparar las tendencias dermatoglíficas digitales que como población caracteriza a los zoques, en el marco de la variación de dichos rasgos papilares de los grupos indígenas de Chiapas y otras poblaciones mesoamericanas.

En el cuadro 6 se han reunido las frecuencias de dermatoglifos digitales y el porcentaje de deltas reportados en la literatura para tales grupos indigenas, en lo que se refiere al sexo masculino. ${ }^{2}$ En el caso de Chiapas, son los tzeltales y tzotziles los únicos pueblos indigenas estudiados desde el punto de vista dermatoglífico. La comparación debe pues, extenderse a otros grupos mayances y aun a los del centro dé México, con el fin de situar más convenientemente al grupo zoque.

La caracteristica dermatoglifica más notable de los pueblos indí-

${ }^{3}$ Los datos publicados sobre series femeninas de dermatoglifos son insuficientes para intentar un examen comparativo. 
genas de los Altos de Chiapas, según ha sido reportado (Newman, 1960; Coope y Roberts, 1971) es el alto porcentaje de torbellinos, que se traduce en un índice de intensidad de patrón también elevado. En cambio los mayas de Yucatán y los pueblos de habla mayance de Guatemala, según Newman (1960) presentan valores de este índice más bajos, lo cual permitiría establecer una frontera determinada por el valor de 13.4; cifras mayores a ésta, caracterizarían

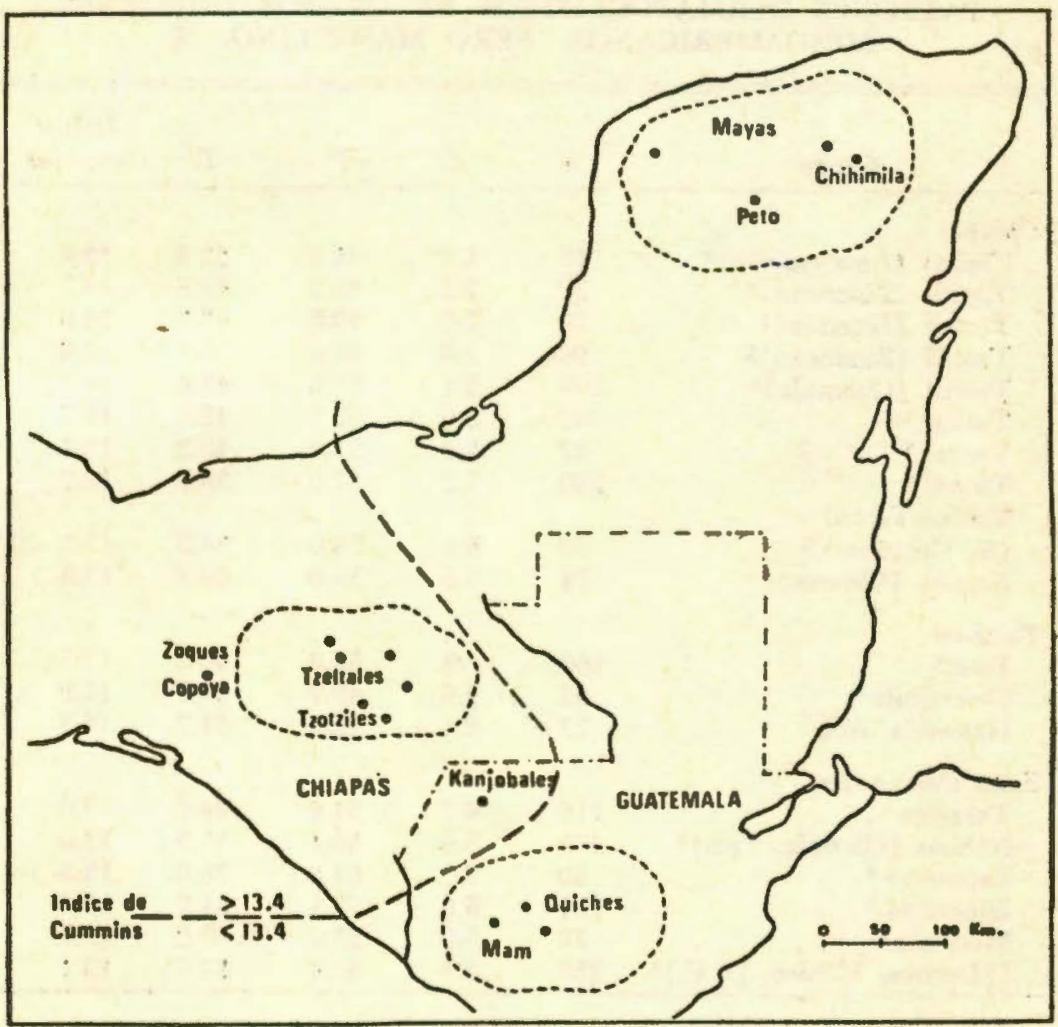

Froura 2. El Indice de Cummins en el área maya. (Según Newman, 1960). (Se han agregado algunas localidades estudiadas reciientemente).

a los pueblos de los Altos de Chiapas; menores, a los pueblos mayas que habitan en Yucatán y Guatemala (Fig. 2).

Sin embargo, datos más recientes (Serrano, 1973), indican que los mayas de Yucatán alcanzan frecuencias de torbellinos elevadas, superiores al $40 \%$, con índices de intensidad de patrón que rebasan también la cifra antes mencionada de 13.4.

En efecto, si examinamos los valores porcentuales de los dermato- 
glifos digitales y el índice de Cummins en muestras indígenas de Chiapas y Yucatán (cuadro 6) se observa que la frecuencia de torbellinos es superior al $40 \%$ (con excepción de dos series de tzeltalestzotziles, entre un total de 9), con un índice de Cummins superior a 13.4. Esta situación equipara entonces a los grupos de Chiapas

$$
\text { Cuadro } 6
$$

PATRONES DERMATOGLIFICOS EN GRUPOS INDIGENAS MESOAMERICANOS. SEXO MASCULINO. \%

\begin{tabular}{|c|c|c|c|c|c|}
\hline Grupo & $n$ & $A$ & $P$ & $T$ & $\begin{array}{c}\text { Indice } \\
\text { Cummins }\end{array}$ \\
\hline \multicolumn{6}{|l|}{ Chiapas } \\
\hline Tzeltal (Amatenango) ${ }^{1}$ & 49 & 1.2 & 46.4 & 52.4 & 15.4 \\
\hline Tzotzil (Zinancan) ${ }^{1}$ & 24 & 2.2 & 48.2 & 49.6 & 14.7 \\
\hline Tzotzil (Huixtán) ${ }^{1}$ & 50 & 2.5 & 49.6 & 48.9 & 14.6 \\
\hline Tzotzil (Zinancan $)^{1}$ & 90 & 2.8 & 46.8 & 50.4 & 14.8 \\
\hline Tzotzil (Chamula) ${ }^{1}$ & 100 & 3.4 & 52.8 & 43.8 & 14.0 \\
\hline Tzeltal 1 & 62 & 5.0 & 52.6 & 42.4 & 13.7 \\
\hline Tzeltal 1 & 47 & 2.8 & 57.0 & 40.2 & 13.7 \\
\hline Tzotzil 1 & 230 & 7.2 & 57.0 & 35.8 & 12.9 \\
\hline $\begin{array}{l}\text { Tzeltal-Tzotzil } \\
\text { (S Cristóbal) } 2\end{array}$ & & & & & \\
\hline (S. Cristóbal) 2 & 90 & 6.4 & 58.6 & 34.9 & 13.5 \\
\hline Zoques (Copoya) ${ }^{3}$ & 74 & 6.6 & 53.0 & 40.3 & 13.5 \\
\hline \multicolumn{6}{|l|}{ Yucatán } \\
\hline Peto 4 & 160 & 4.9 & 54.8 & 40.3 & 13.5 \\
\hline Chichimilá & 62 & 5.6 & 49.7 & 44.7 & 11.9 \\
\hline Hacienda Acú ${ }^{1}$ & 25 & 6.4 & 42.4 & 51.2 & 14.5 \\
\hline \multicolumn{6}{|l|}{ Zona Centro-Sur } \\
\hline Tarascos 1 & 116 & 4.2 & 61.6 & 34.2 & 13.0 \\
\hline Náhuas (Cholula, Pue.) ${ }^{5}$ & 178 & 5.6 & 58.1 & 35.3 & 13.0 \\
\hline Zapotecos ${ }^{1}$ & 50 & 3.0 & 61.0 & 36.0 & 13.3 \\
\hline Zapotecos 2 & 104 & 6.6 & 59.1 & 34.2 & 13.3 \\
\hline Mixtecos 1 & 78 & 3.0 & 57.0 & 40.0 & 13.7 \\
\hline (Mestizos, México, D. F.)6 & 250 & 3.4 & 61.4 & 32.6 & 13.1 \\
\hline
\end{tabular}

1 Datos recopilados por Coope y Roberts, 1971.

2 Zavala y col., 1971.

3 Presente estudio.

4 Serrano, 1973.

5 Serrano, 1971.

6 Zavala y col., 1969.

con los mayas yucatecos y los distingue de las poblaciones del centrosur de México, que presentan, de acuerdo a los datos hasta ahora conocidos, frecuencias de torbellinos menores de $40 \%$ y valores del índice de Cummins también más reducidos.

Esta situación se evidencia gráficamente en la figura 3, donde 
se muestra la distribución de diferentes series mesoamericanas en relación a la frecuencia de presillas y torbellinos. Se puede observar que el límite de $40 \%$ de torbellinos separa a las series del sureste de las del centro-sur de México.

¿Qué posición guarda la población zoque dentro de este campo de variabilidad?

Tanto su frecuencia de torbellinos de $40.4 \%$ como el índice de Cummins, igual a 13.4 los coloca en el límite inferior de la variación

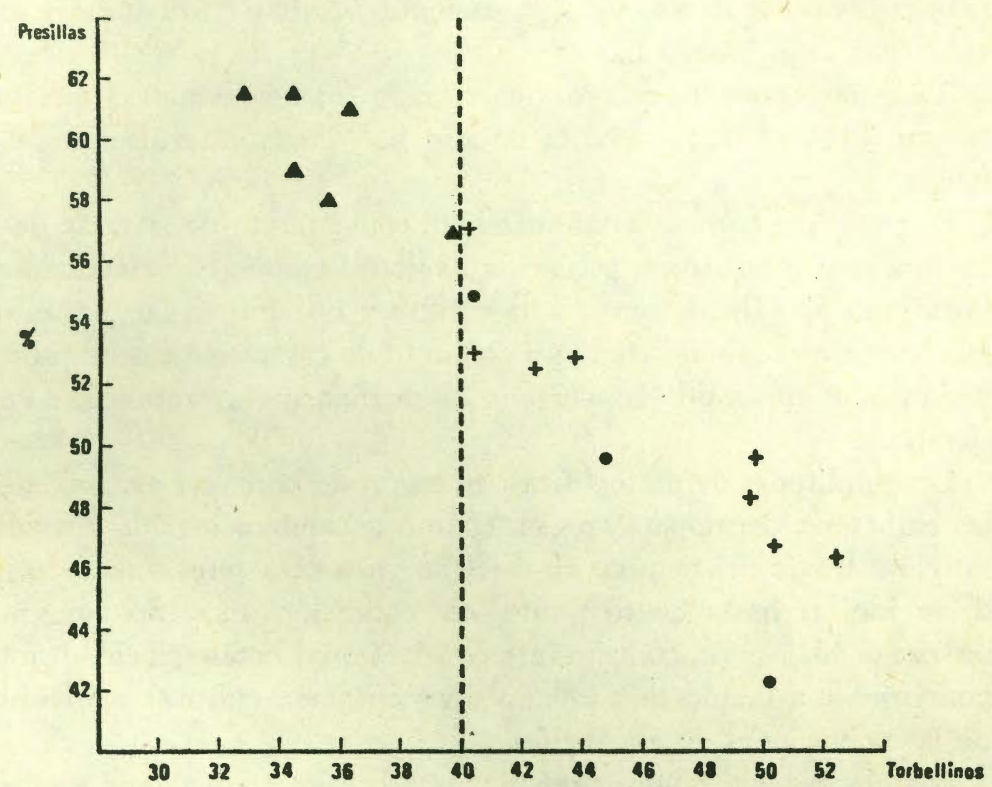

Frgura 3. Distribución de poblaciones indígenas mesoamericanas de acuerdo a frecuencia de Presillas Torbellinos \%.

+ Tzeltales-tzotziles.

- Mayas yucatecos.

$\Delta$ Grupos indígenas de la zona centro-sur de México.

dermatoglífica de los pueblos mayas. En la figura 3, la serie zoque se sitúa así junto al limite establecido entre los dos mencionados grupos de poblaciones, pero en más estrecha contigüidad con los pueblos mayas.

Es necesario, por supuesto, el examen de otros datos dermatoglíficos, que abarquen además un número mayor de poblaciones mesoamericanas. Las series del altiplano y sur de México hasta ahora son muy exiguas, y en el caso del sureste únicamente se han estudiado mayas 
y tzeltales-tzotziles. Sólo una mayor información podría confirmạr o precisar las observaciones aquí establecidas.

Por otra parte cabría preguntarse hasta qué punto es posible, con base en los resultados obtenidos, explicar éstos en función de una antigua comunidad de origen de los pueblos del sureste de México entre los cuales estarían incluidos los zoques. Dadas las característicsa genéticas de los dermatoglifos: herencia poligénica, y por lo tanto no fácilmente sensible a la deriva genética; independencia postnatal de influencias ambientales, etc., las semejanzas entre las características dermopapilares entre poblaciones podrían quizá indicar antiguas relaciones biológicas.

A este respecto cabe recordar el llamado "modelo genético" propuesto por Vogt (1971), para el estudio del desarrollo cultural de los mayas.

Este modelo considera teóricamente como punto de partida de los pueblos mayances a una población ancestral común caracterizada por un mismo tipo físico, una misma cultura y una misma lengua, el primer elemento definido como un conjunto de características que se asemejan más, en sentido convergente, a medida que retrocedemos en el tiempo.

Las similitudes dermatoglíficas, en razón de la mayor estabilidad de los caracteres dermopapilares en cuanto a cambios genéticos o adaptativos a través del tiempo, vis-à-vis de otros caracteres somáticos, podrían indicar hasta cierto punto, esa condición ancestral común de los rasgos biológicos, contrastante con la actual heterogeneidad antropométrica o a la aun más intensa diversificación cultural y lingüística de las poblaciones de esa región.

Las observaciones aquí presentadas sobre los zoques, que se sitúan en el campo de variación dermatoglífica conocida de los grupos mayances (aunque cercanos también a otros grupos mesoamericanos nomayances), parecen encaminarse en ese sentido.

Se trata, no obstante de una interpretación preliminar acerca de una hipótesis que precisa de un análisis más amplio, en el cual el estudio dermatoglífico se revela como un útil procedimiento metodológico que puede coadyuvar al esclarecimiento de ese problema.

\section{REFERENCIAS}

Coope, E. Y D. F. Roherts

1971 "Dermatoglyphic studies of populations in Latin America . The ongoing evolution of Latin American Populations, ed. F. M. Salzano, pp. 405-53, Charles C. Thomas, Publ. Springfield. 
Cummins, H. у C. Midlo

1961 Finger Prints, Palms and Soles. Dover, New York.

Matson, G. A.

1970 "Distribution of Blood Groups . Handbook of Middle Amsrican Indians, Physical Anthropology, 9: 105-147, Austin.

Navarrete, C.

1970 "Fuentes para la historia cultural de los zoques". Anales de Antropología, 7: 207-246, Instituto de Investigaciones Históricas, UNAM, México.

Newman, M. T.

1960 "Populational analysis of finger and palm prints in Lowland Maya Indians". American Journal Physical Anthropology. 18: 45-58.

1970 "Dermatoglyphics". Handbook of Middle American Indians, Physical Anthropology, 9: 167-79, Austin.

Pons, J.

1963 "An evaluation of the usefulness of dermatoglyphics in research". Roma, Proceedings Internactional Congress Human Genetics, 111: 1458-69.

Rife, D. C.

1953 "Finger prints as criteria of ethnic relationship". American Journal Human Genetics, 5: 389-99.

Serrano, C.

1971 "Los dematoglifos digitales en la población masculina de Cholula, Pue.". Anales del Instituto Nacional de Antropología e Historia, T. 2, 7a. Ep., pp. 59-66, México.

1973 "Los dermatoglifos digitales en dos poblaciones mayas de Yucatán". Estudios de Cultura Maya, 9: 17-28, Centro de Estudios Mayas, UNAM, México.

StARR, F.

1902 "The physical characters of the indians of Southern Mexico". Decennial Publications, 4: 63-109, University of Chicago.

Swadesh, M.

1960 Estudios sobre Lengua y Cultura. Acta Anthropologica, 2a. Ep., 11-2, Escuela Nacional de Antropología, México.

Thomas, N. D.

1974 'The linguistic, geographic, and demographic position of the Zoque of Southern Mexico". Papers of the New World Ar-

VoGT, E. Z. chaelogical Foundation, No. 36, Provo.

1971 "The genetic model and Maya Cultural development". De sarrollo Cultural de los Mayas, E. Z. Vogt y A. Ruz, Eds., pp. 9-48. Centro de Estudios Mayas, UNAM, México.

Zavala, G., A. Cово y R. Lisker

1969 "Dermatoglyphic Patterns in a Sample of Normal Urban Mexicans". Human Heredity, 19: 534-539.

1971 "Dermatoglyphic Patterns in Mexican Indian Groups". Human Heredity, 21: 394-401. 